\title{
Using a Variability Factor to Account for Cloud Microphysical Inhomogeneity in Mesoscale Models
}

\author{
YEFIM KOGAN \\ NorthWest Research Associates, Redmond, Washington
}

(Manuscript received 10 October 2017, in final form 25 April 2018)

\begin{abstract}
Different formulations of the joint probability distribution function (JPDF) based on large-eddy simulation (LES) studies of shallow cumulus and cumulus congestus clouds were evaluated. It was shown that inhomogeneity in both cloud types can be quantified by their respective JPDFs calculated using datasets from the entire simulation time period ("generic" JPDFs). The generic JPDF can be a priori integrated and yield a one-dimensional variability factor ( $V$ factor) specific for each cloud type. A quite accurate approximation of $V$ factors by an analytical function in the form of a third-order polynomial was obtained and can be easily implemented in mesoscale models. The effect on precipitation of conversion rates modified by $V$ factors was also evaluated in LES sensitivity studies of shallow cumulus $(\mathrm{Cu})$ and congestus $\mathrm{Cu}$ clouds. The surface precipitation increased significantly when $V$ factors were taken into account. The sensitivity experiments revealed that most of the increase resulted from the modified autoconversion process. The effect of accretion rates modified by $V$ factors was much less significant, primarily because of the nearly linear dependence of accretion on its parameters. This fact shows the importance of the most accurate formulation of the autoconversion process.
\end{abstract}

\section{Introduction}

Neglecting subgrid-scale (SGS) variability in mesoand large-scale models can lead to substantial bias in calculations of microphysical process rates (Pincus and Klein 2000; Larson et al. 2001a; Wood et al. 2002). Many studies addressed the microphysical inhomogeneity problem by using variety of observational, theoretical, and modeling techniques. The detailed discussion of these approaches is given in Kogan and Mechem (2014, 2016, hereafter KM14 and KM16); here, we present just a brief summary.

The most common method to calculate unbiased process rates is to integrate them over the mesoscale model grid by using SGS probability distribution functions (PDFs) of corresponding microphysical variables. For process rates that are a function of multiple variables, we need the knowledge of the joint probability distribution function (JPDF) of the different variables. Previous studies have established PDFs of these variables from observations (e.g., Price and Wood 2002; Lee et al. 2010; Larson et al. 2001a,b), numerical models

\footnotetext{
Corresponding author: Yefim Kogan, ykogan@nwra.com
}

(Xu and Randall 1996; Wang and Stevens 2000), or theoretical considerations (Lappen and Randall 2001; Lewellen and Yoh 1993; Golaz et al. 2002; Cheng and Xu 2006, 2009). Perhaps the most sophisticated approach employs both observational and modeling data to formulate joint analytic PDFs of liquid water potential temperature, total water, and vertical velocity [Cloud Layers Unified by Binomials (CLUBB); Larson et al. 2002; Golaz et al. 2002; Larson and Golaz 2005]. Under the CLUBB approach, theoretical considerations are used to establish the shape of the distributions with parameters constrained from large-eddy simulation (LES) output and aircraft observations.

KM14 and KM16 proposed a method that establishes JPDFs from a series of large-eddy simulations of precipitating convective cloud systems. Their methodology includes two distinct elements for deriving the JPDF parameterization. The first is the use of a layered approach to calculate JPDFs; this increases the statistical robustness of the JPDFs. The second element is the formulation of the JPDFs in nondimensional space by normalizing the microphysical variables by their horizontal-layer-mean values. The JPDFs for autoconversion and accretion processes were obtained by 
analyzing LES data from two cases. In the first case, shallow cumulus $(\mathrm{Cu})$ clouds were simulated based on data from the Rain in Cumulus over the Ocean (RICO; Rauber et al. 2007) field campaign. In the second case, a more vigorous congestus cloud system was simulated based on data obtained during the Tropical Ocean and Global Atmosphere Coupled Ocean-Atmosphere Response Experiment (TOGA COARE; Godfrey et al. 1998) field project.

Both of these cloud systems constitute two distinct modes of trade wind tropical cloudiness that produce a substantial amount of tropical precipitation with estimates ranging from $20 \%$ to $\sim 32 \%$ (Cheng and Houze 1979; Stephens and Wood 2007; Liu and Zipser 2009). Mechem and Oberthaler (2013) summarize some of the methodological differences behind these different estimates and find a congestus contribution of $34 \%$ based on simulations from a 10-day period during TOGA COARE.

KM14 and KM16 have tested a number of JPDF approximations of various degrees of complexity. The present study further advances these approximations by developing a new efficient formulation of SGS variability that can be easily implemented into mesoscale models.

\section{Model and dataset}

The model employed in our simulations is a version of the Cooperative Institute for Mesoscale Meteorological Studies (CIMMS) LES (Kogan et al. 1995; Khairoutdinov and Kogan 1999) called System for Atmospheric Modeling (SAM)-Bulk Microphysics (SAMBM; Khairoutdinov and Kogan 2000; Kogan 2013). The SAM (Khairoutdinov and Randall 2003) forms the dynamical core of SAMBM. SAM is based on anelastic dynamics and monotonic, positive-definite advection for scalars (Smolarkiewicz and Grabowski 1990). The bulk microphysical parameterization (Kogan 2013) employed in SAMBM was developed based on drop size distribution output from the CIMMS LES model with explicit (bin) microphysics (SAMEX; Kogan et al. 2012).

Shallow cumulus clouds were simulated based on data from the RICO field campaign (vanZanten et al. 2011). The integration domain was $25.6 \times 25.6 \times 4.0 \mathrm{~km}^{3}$

\footnotetext{
${ }^{1}$ Depending on the size and resolution of the simulated meteorological phenomena, mesoscale models may use horizontal grids in the 5-50-km range. The dependence of JPDFs on the LES domain size (which mimics a mesoscale model grid) was studied in the RICO case with results shown in the appendix.
}

using a horizontal resolution of $100 \mathrm{~m}$ and a vertical resolution of $40 \mathrm{~m}$. The analysis dataset was selected from clouds evolving during the second half of the 24-h simulation when deeper clouds appear and precipitation is most intense. The $25.6-\mathrm{km}$ horizontal domain size in RICO simulation roughly corresponded to the grid size of an NWP model. ${ }^{1}$

For cumulus congestus, we used the initial profiles, large-scale vertical velocity, and tendencies of temperature and moisture corresponding to conditions of day 344 during the TOGA COARE field campaign. The horizontal grid spacing for the simulation was $100 \mathrm{~m}$; in the vertical, a stretched grid was used as described in KM16. The total of $384 \times 384 \times 130$ grid points was used for the domain size of $38.4 \times 38.4 \times 11 \mathrm{~km}^{3}$. To attribute precipitation to clouds of different thickness, KM14 and KM16 divide the dataset into four groups based on quartiles of the cumulative cloud-top distribution. The top quartile [group 4 (G4)] in the TOGA dataset included a mixture of clouds with tops in the range from 2.7 to $7 \mathrm{~km}$. G4 in the RICO case contains clouds with tops in the narrower range, from 2.46 to $3 \mathrm{~km}$, which makes physical interpretation of variability in the RICO case much easier. As the precipitation contribution from the G4 clouds is substantially larger than that from other cloud categories, we concentrate in our analysis on clouds in G4.

\section{Effect of different JPDF approximations on conversion rates}

We focus on two JPDFs that are used in calculations of microphysical autoconversion and accretion rates. The first is used for calculation of autoconversion; it depends on cloud drop number concentration $N_{c}$ and cloud water mixing ratio $q_{c}$. The second is needed for calculation of accretion; it depends on $q_{c}$ and rainwater mixing ratio $q_{r}$. In general, the JPDFs at each mesoscale model grid point depend on the grid averages of resolvable prognostic variables. The analytical formulation of such dependencies (closure schemes) is a complex task; a more modest approach is to use simplified approximations of JPDFs. In this study, we evaluate the errors introduced by such approximations and propose a simple formulation of subgrid variability for calculations of autoconversion and accretion (conversion) rates over the mesoscale model grid.

As was shown in KM14 and KM16, the JPDFs significantly change in the vertical and depend on parameters characterizing cloud system environment. In the case of our simulations, vertical dependence can be studied explicitly, while various environmental conditions are created as simulation evolves in time. 
The detailed description of cloud-system thermodynamical parameters in RICO case can be found, for example, in vanZanten et al. (2011); more microphysical details are discussed in Kogan et al. (2012). Mechem and Oberthaler (2013) describe dynamical and microphysical parameters of the congestus cumulus TOGA COARE case. As our focus is on parameterization of cloud microphysical variability, we show in Fig. 1 two parameters characterizing cloud microstructure: liquid water path (LWP) and the rainwater path (RWP), both averaged over the "cloudy columns" (defined as columns with LWP $>0.01 \mathrm{~g} \mathrm{~m}^{-2}$ ). Also shown is horizontally averaged and vertically integrated turbulent kinetic energy (TKE); it illustrates the extent of dynamical changes in the cloud system as a whole. ${ }^{2}$

The LWP in the RICO case is highly variable and increased over the course of the last $12 \mathrm{~h}$ by $60 \%$. The precipitation intensifies as clouds mature, so RWP over the same period increased sixfold. In the TOGA COARE case, the more frequent shallow cumulus clouds were mixed with powerful cumulus congestus; the latter contribute most to precipitation [see Mechem and Oberthaler (2013) and references therein]. The variability of microphysical parameters in the TOGA COARE simulation was even more dramatic. For instance, LWP varied in the $60-800 \mathrm{~g} \mathrm{~m}^{-2}$ range and RWP in the $0-500 \mathrm{~g} \mathrm{~m}^{-2}$ range. The variation in TKE correlated well with precipitation activity and was especially pronounced in the TOGA COARE simulation.

Clearly, the hourly datasets, which we used for calculation of time-dependent JPDFs, varied quite significantly and represented a wide range of environmental conditions. We also considered a case where time dependence and, therefore, varying environmental conditions were neglected. These JPDFs were calculated using the dataset from the whole simulation; they are referred to as "generic" JPDFs. Employing these two formulations and the layer-mean values of $q_{c}, N_{c}$, and $q_{r}$, we calculated conversion rates, which are referred to as "approximated" conversion rates. The approximated rates were compared with the "benchmark" conversion rates, which were obtained using the values of $q_{c}, N_{c}$, and $q_{r}$ at each LES grid point and then averaged for each layer. ${ }^{3}$

Finally, we also considered an approximation when conversion rates were calculated using only the layer-

\footnotetext{
${ }^{2}$ To show all parameters on the same plot, the TKE is scaled by a factor indicated in the Fig. 1 caption.

${ }^{3}$ More details on calculations of approximated and benchmark conversion rates can be found in KM14.
}
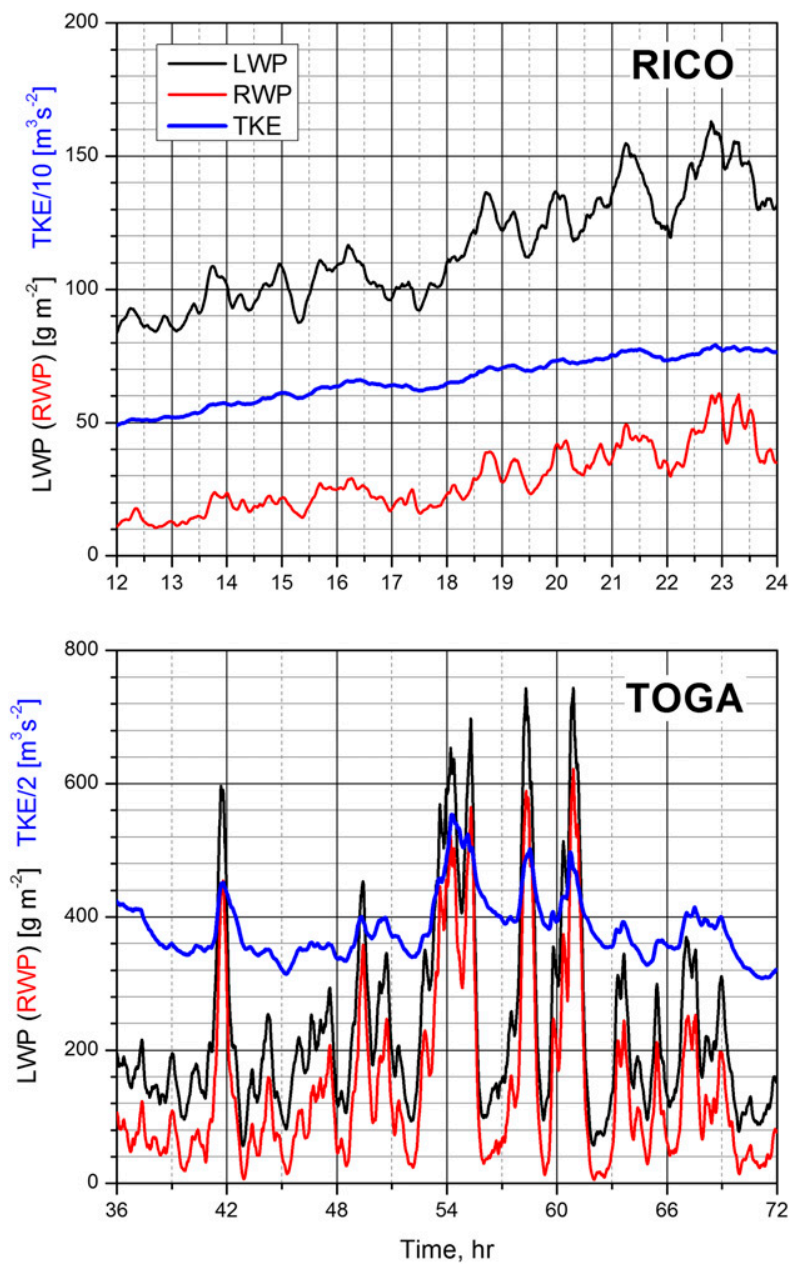

FIG. 1. The evolution of LWP, RWP, and TKE during the (top) RICO and (bottom) TOGA COARE simulations. TKE values were multiplied by factor $f=0.1$ in the RICO case and $f=0.5$ in the TOGA COARE case.

mean variables; that is, JPDFs were neglected altogether. This noPDF case was used to evaluate the decrease in errors when time-dependent or generic JPDFs were accounted for in calculations of conversion rates.

The errors of different approximations were defined as

$$
\varepsilon=(A / B-1) \times 100,
$$

where $A$ and $B$ denote approximated and benchmark conversion rates, respectively. The errors were calculated for each vertical layer and each hour of simulation, that is, for the last $12 \mathrm{~h}$ of the RICO and the last $36 \mathrm{~h}$ of the TOGA COARE case. Figure 2 shows the cumulative distribution of the errors for autoconversion and accretion rates in the RICO case. The smallest errors were produced by JPDFs that vary both in time and in 

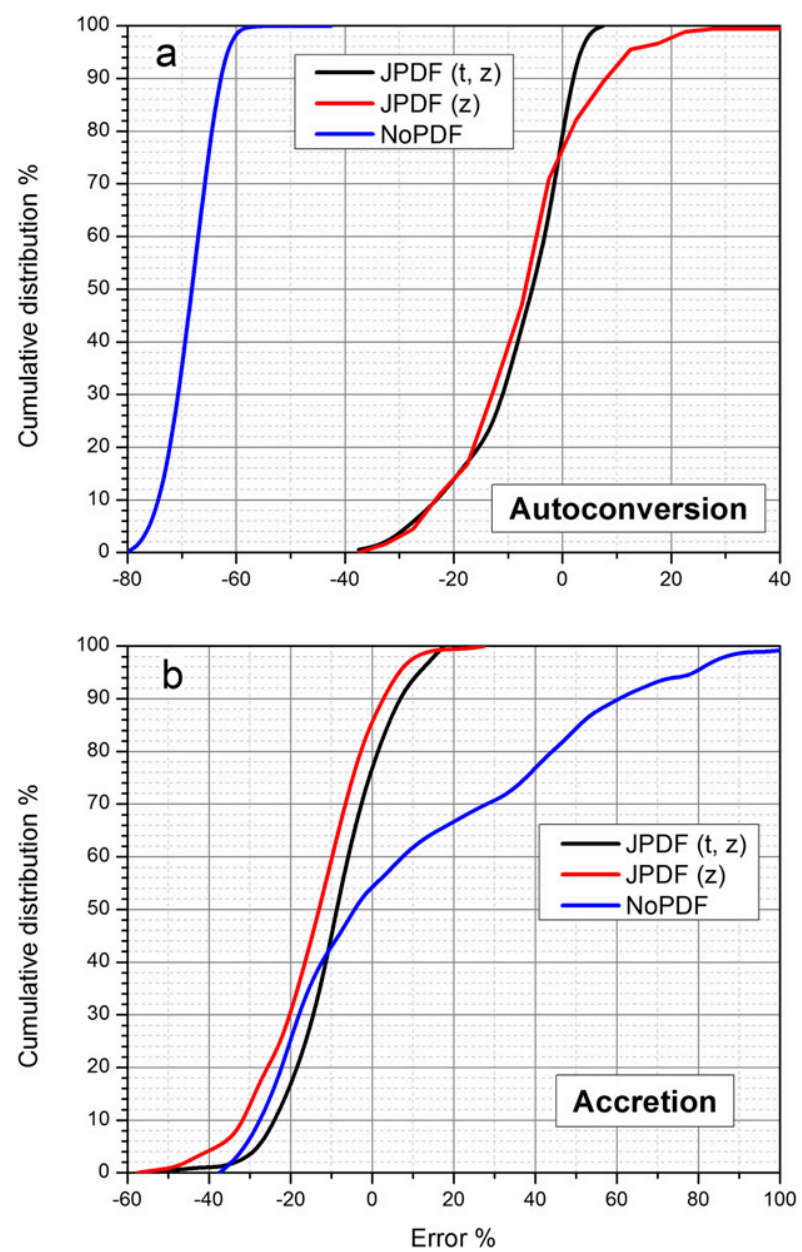

FIG. 2. The cumulative distributions of errors in the RICO case using different JPDF approximations in calculations of (a) autoconversion and (b) accretion rates. The black curve represents rates approximated by a JPDF varying in time and in the vertical. The red curve represents the case with the generic (independent of time) but height-dependent JPDF. The blue curve refers to a calculation when SGS variability is neglected.

the vertical (Fig. 2, black curve). Although the timedependent JPDFs were tuned each hour to the clouds they represent, nevertheless, the conversion rates still show errors. This is because of the use of rather coarse resolution in discretizing the JPDFs (only $15 \times 15$ bins and limiting the variability of dimensionless variables to the range from 0 to 3 ).

Remarkably, the errors of the generic JPDF were not significantly larger than the errors from the timedependent JPDF (red curve in Fig. 2). The most noticeable difference is for the upper quantile of the data where generic JPDF had slightly larger errors. These errors, nevertheless, were significantly smaller than errors in the noPDF case. Neglecting SGS variability
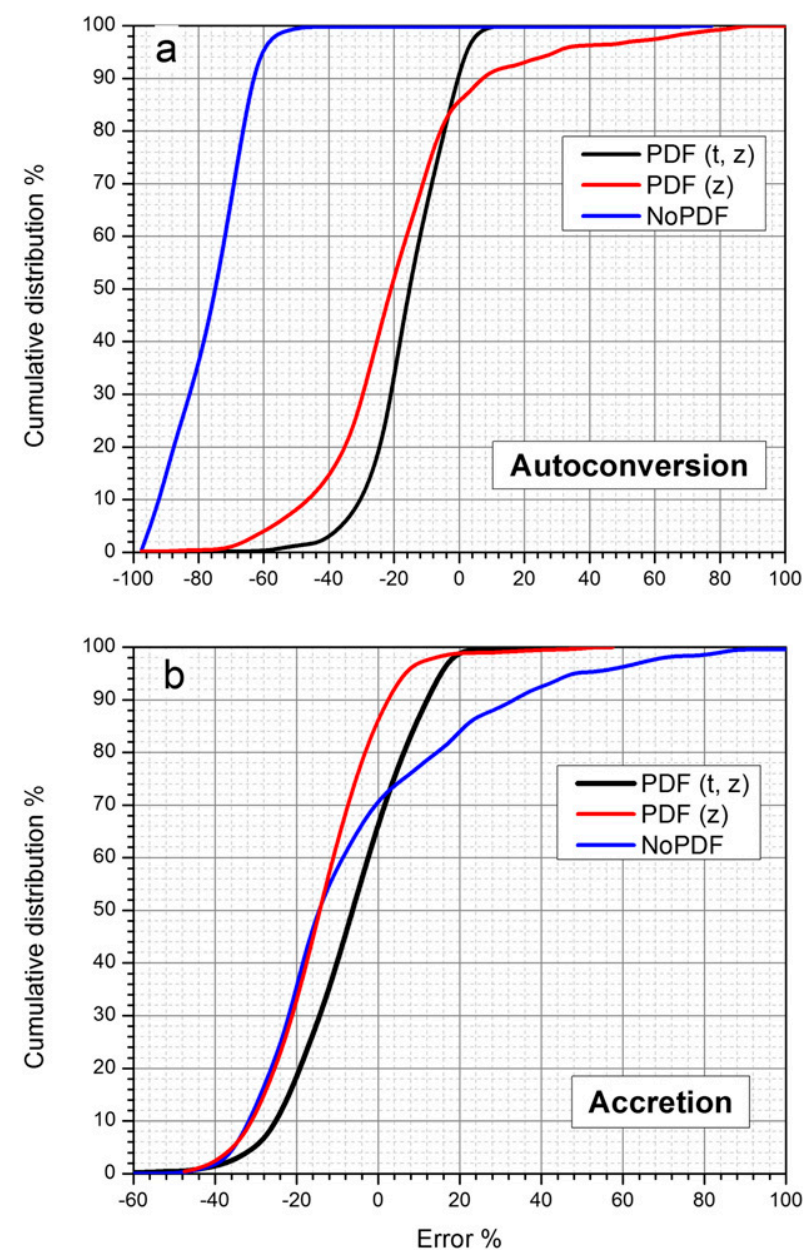

FIG. 3. As in Fig. 2, but for the TOGA COARE case.

introduced substantial negative bias for autoconversion rates with errors in the $-60 \%$ to $-80 \%$ range.

The accretion errors were even less sensitive to the choice of JPDF (Fig. 2b). The difference in accretion errors between the time-dependent and generic JPDF is rather small; namely, the use of generic JPDF increased the negative bias only by a few percent. Totally neglecting variability (noPDF case) resulted in more significant errors of both signs. However, while the negative errors did not exceed more than $-40 \%$, the positive errors varied in a wider range with a tail extending to about $100 \%$.

In the TOGA COARE case, the generic autoconversion errors were larger but essentially followed the same pattern as in RICO case (Fig. 3a). About $15 \%$ of the data had positive errors that are slightly higher compared to the RICO case; for the rest of the data, the errors were negative. Neglecting SGS variability altogether introduced a significant negative bias (in the $-80 \%$ to $-100 \%$ range) for all data. 
The pattern of the bias for generic accretion rates was similar to the RICO case; however, the errors were slightly higher (Fig. 3b). Totally neglecting variability, in addition to negative bias similar to the generic case, also introduced a considerable positive bias with errors up to $100 \%$.

Figures 2 and 3 reveal only the overall distribution of errors; in the section below, we also show the vertical distribution of errors, as well as their dependence on the value of the rate itself. Such information is helpful in interpretation of the effect of inhomogeneity on precipitation.

The main focus of this section was on comparison of the most accurate time-dependent JPDF with its generic counterpart. Our LESs showed that, although generic JPDFs are less accurate then the time-dependent JPDFs, they nevertheless present a significant improvement compared to the case when SGS variability is totally neglected. In the next section, we propose a simple method to implement generic JPDFs in mesoscale models by introducing the variability factor.

\section{Formulation of the variability factor}

We formulate JPDFs in terms of dimensionless parameters $\phi, \psi$, and $\zeta$ defined as

$$
\varphi=q_{c} / \overline{q_{c}}, \quad \psi=N_{c} / \overline{N_{c}}, \quad \zeta=q_{r} / \overline{q_{r}},
$$

where $\overline{q_{c}}, \overline{N_{c}}$, and $\overline{q_{r}}$ are horizontal averages of $q_{c}, q_{r}$, and $N_{c}$ over the cloudy areas at each layer.

The local autoconversion rate, as defined on a small LES grid cell, is a highly nonlinear function of its two parameters (e.g., Khairoutdinov and Kogan 2000; Seifert and Beheng 2001; Kogan 2013). In trade wind shallow cumulus, it may be expressed as (Kogan 2013)

$$
\left(\frac{\partial q_{r}}{\partial t}\right)_{\text {auto }}=7.98 \times 10^{10} q_{c}^{4.22} N_{c}^{-3.01} .
$$

The autoconversion rate over the mesoscale model grid should take into account SGS variability of microphysical variables. Therefore, it should integrate local rates weighed by the JPDF of microphysical variables $q_{c}$ and $N_{c}$. As a result, the mesoscale grid average autoconversion rate is expressed as

$$
\begin{aligned}
\bar{R}_{\text {auto }} & =\overline{C_{\text {au }} q_{c}^{\alpha} N_{c}^{\beta}} \equiv \iint C_{\text {au }} q_{c}^{\alpha} N_{c}^{\beta} \Omega\left(z, q_{c}, N_{c}\right) d q_{c} d N_{c} \\
& =\left.C_{\text {au }}\left(\overline{q_{c}}\right)^{\alpha}\left(\overline{N_{c}}\right)^{\beta} \iint \varphi^{\alpha} \psi^{\beta} \Omega^{\prime}\right|_{\overline{q_{c}} \overline{N_{c}}}(z, \varphi, \psi) d \varphi d \psi .
\end{aligned}
$$

Here, $\Omega$ is the JPDF of variables $q_{c}$ and $N_{c}$, and $\Omega^{\prime}$ is its nondimensional analog in the $\varphi-\psi$ space defined from relationship

$$
\Omega^{\prime}(z, \varphi, \psi) d \varphi d \psi=\Omega\left(z, q_{c}, N_{c}\right) d q_{c} d N_{c} .
$$

Following the findings in KM14 and KM16 that showed significant differences between JPDFs at different vertical layers, we indicate in (4) the dependence of $\Omega$ and $\Omega^{\prime}$ on $z$ explicitly. With the values of exponents in (3) $\alpha \approx 4.2$ and $\beta \approx-3$, we can expect substantial difference between the exact expression given by (4) and approximation based on grid-averaged variables that neglects variability of microphysical parameters:

$$
\bar{R}_{\text {auto }} \approx C_{\text {au }}\left(\overline{q_{c}}\right)^{\alpha}\left(\overline{N_{c}}\right)^{\beta} .
$$

Introducing the variability factor ( $V$ factor) $V_{\text {au }}$,

$$
V_{\mathrm{au}}\left(z, \overline{q_{c}}, \overline{N_{c}}\right)=\left.\iint \varphi^{\alpha} \psi^{\beta} \Omega^{\prime}\right|_{\overline{q_{c}} \overline{N_{c}}}(z, \varphi, \psi) d \varphi d \psi,
$$

we can rewrite (4) as

$$
\bar{R}_{\text {auto }}=C_{\text {au }}\left(\overline{q_{c}}\right)^{\alpha}\left(\overline{N_{c}}\right)^{\beta} V_{\text {au }}\left(z, \overline{q_{c}}, \overline{N_{c}}\right) .
$$

While, in general, the $V$ factor is a function of gridmean microphysical variables $\overline{q_{c}}$ and $\overline{N_{c}}$ and the vertical coordinate $z$, in the case of generic JPDF, the dependence of $\Omega^{\prime}$ on grid-mean microphysical variables $\overline{q_{c}}$ and $\overline{N_{c}}$ is neglected and, consequently, the JPDF as well as the $V$ factor is a function of $z$ only.

In this case, it can be rewritten as

$$
V_{\mathrm{au}}(z)=\iint \varphi^{\alpha} \psi^{\beta} \bar{\Omega}^{\prime}(\varphi, \psi) d \varphi d \psi,
$$

where generic JPDF $\bar{\Omega}^{\prime}$ is calculated using the dataset obtained from the entire simulation. The double integral can be numerically integrated to yield a $V$ factor as onedimensional function of $z$.

The autoconversion rate [(4)] using generic JPDF is then determined from the expression

$$
\bar{R}_{\text {auto }}=C_{\text {au }}\left(\overline{q_{c}}\right)^{\alpha}\left(\overline{N_{c}}\right)^{\beta} V_{\text {au }}(z) .
$$

Accounting for inhomogeneity, consequently, is reduced to a simple multiplication of the autoconversion rate by the $V$ factor.

The expression for the accretion rate has a functional form similar to the one for autoconversion but involves $q_{c}$ and $q_{r}$ :

$$
\left(\frac{\partial q_{r}}{\partial t}\right)_{\mathrm{accr}}=C_{\mathrm{ac}} q_{c}^{\gamma} q_{r}^{\delta}=8.53 q_{c}^{1.05} q_{r}^{0.98} .
$$


The difference is that exponents $\gamma$ and $\delta$ in the formulation of the accretion rate are close to 1 , which makes the dependence [(11)] nearly linear; therefore, the effects of inhomogeneity may be expected to be less pronounced.

The grid-mean accretion rate is expressed by double integrals as

$$
\bar{R}_{\mathrm{accr}}=\overline{C_{\mathrm{ac}} q_{c}^{\gamma} q_{r}^{\delta}} \equiv \iint C_{\mathrm{ac}} q_{c}^{\gamma} q_{r}^{\delta} \Psi\left(z, q_{c}, q_{r}\right) d q_{c} d q_{r},
$$

where $\Psi$ is the JPDF of variables $q_{c}$ and $q_{r}$. Using the generic JPDF $\overline{\Psi^{\prime}}$, which depends only on $z$, we can rewrite (12) as

$$
\bar{R}_{\mathrm{accr}} \approx C_{\mathrm{ac}}\left(\overline{q_{c}}\right)^{\gamma}\left(\overline{q_{r}}\right)^{\delta} V_{\mathrm{accr}}(z),
$$

where the $V$ factor for accretion is given by the double integral:

$$
V_{\mathrm{accr}}(z)=\iint \varphi^{\gamma} \psi^{\delta} \overline{\Psi^{\prime}}(z, \varphi, \psi) d \varphi d \psi
$$

The $V$ factors in (9) and (14) for autoconversion and accretion rates are shown in Fig. 4 for the RICO and TOGA COARE cases. Their profiles reveal how the errors due to neglect of SGS variability are distributed in the vertical, thus supplementing Figs. 2 and 3, which show only the overall distribution of errors. As noPDF autoconversion errors are always negative, the $V$ factor is larger than 1 in both simulations. For shallow cumulus, the $V$ factor varies in the range 2.5-3 with a slight minimum in the middle of the clouds. As noted in section 2, in the RICO case, the G4 clouds have tops in the rather narrow $2.5-3.1-\mathrm{km}$ range. Therefore, the minimum in the middle of the $V$-factor profile (at about $1.8 \mathrm{~km}$ ) corresponds roughly to the middle of RICO clouds, and the maxima at the bottom and the top correspond, respectively, to cloud base and cloud top.

The bottom maximum reflects the fact that there is more variability at cloud base, where regions of falling raindrops coexist with updrafts regions containing mostly newly formed cloud drops. The regions of falling rain may contain raindrops, which evaporate as they fall. As their size shrinks to less than the threshold size separating cloud and raindrop categories $(r=40 \mu \mathrm{m})$, they are classified as cloud drops. As a result, the falling rain areas also have cloud drops and, thus, contribute to the larger inhomogeneity of $q_{c}$ and $N_{c}$ near cloud base.

Similar levels of variability occur near cloud tops where processes of entrainment of dry air into the cloud and detrainment of cloudy air into the undersaturated
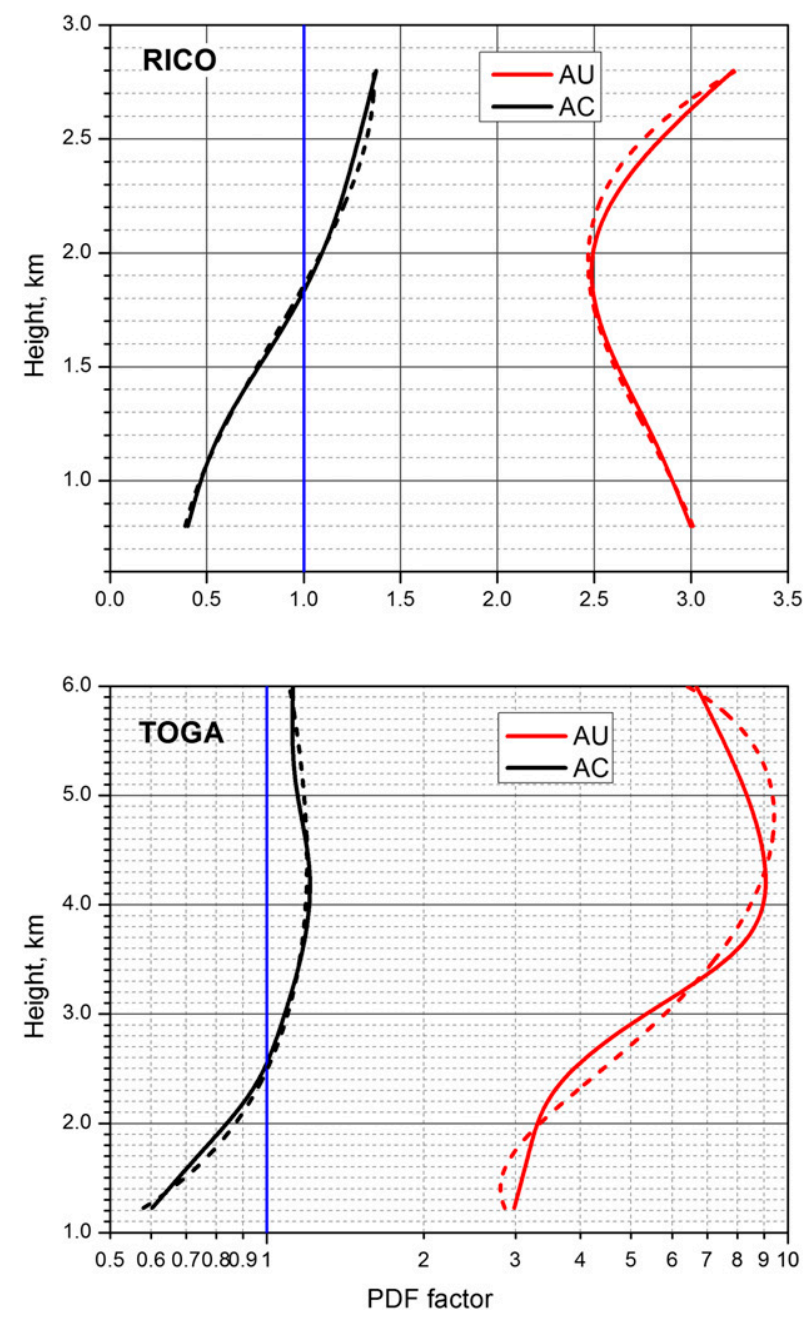

FIG. 4. Variability factor for autoconversion (red) and accretion (black) in (top) RICO and (bottom) TOGA COARE simulations. Third-order polynomial fits are shown by dashed lines.

environment result in evaporation of drops and, therefore, increase the range of drop sizes and concentrations. Wide variation in these parameters results in larger inhomogeneity near cloud top and base.

In the TOGA case, the G4 group contains clouds with tops varying in the much wider $2.7-7-\mathrm{km}$ range. Therefore, the vertical profiles of $V$ factors are statistical averages of data from clouds of different depths. As a result, the vertical coordinate in Fig. $4 \mathrm{~b}$ cannot be uniquely linked to a specific vertical region in a cloud. For instance, the level $z=4 \mathrm{~km}$ may combine data from the middle of a 7-km-tall cloud and the top region of a $4.5-\mathrm{km}$-tall cloud. The obvious exception is the cloudbase region, which is at the same condensation level for all clouds, and where the $V$ factor has the value of about 3 , approximately the same as in RICO clouds. The wide mix of clouds of various depths in the TOGA COARE 

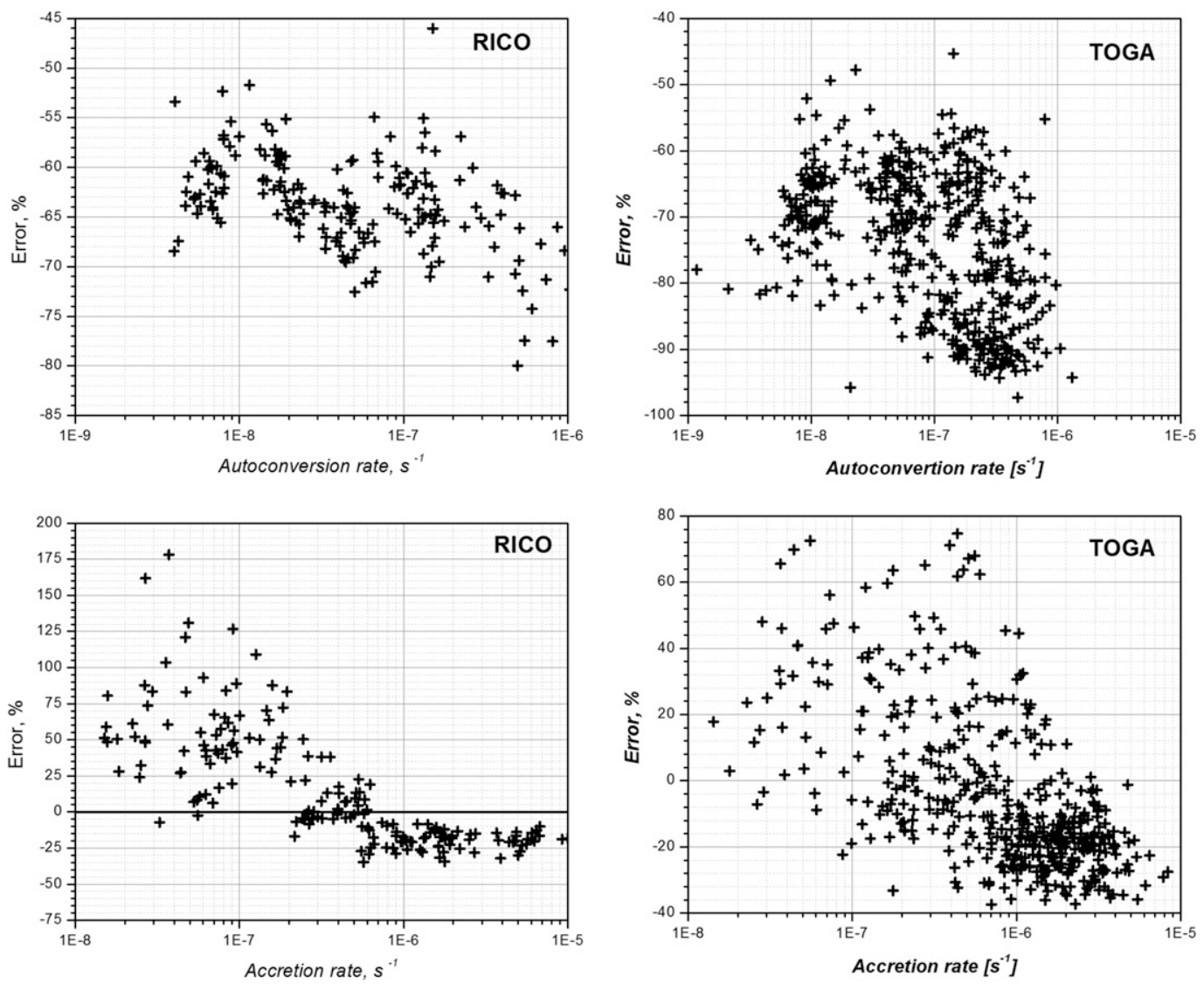

FIG. 5. The dependence of errors on the conversion rate value in RICO and TOGA COARE simulations: (top) autoconversion and (bottom) accretion.

case may explain the difference between autoconversion profiles in both cases; for example, the $V$-factor increase in the TOGA COARE case at about the $4-\mathrm{km}$ level is most likely due to contribution to this level from the more numerous medium-size clouds, with tops in the 4-5-km range. In addition, because of a much wider range of drop sizes and concentrations in the TOGA COARE case, the underestimation of autoconversion is much larger, and the $V$ factor is also larger, varying from 3-4 at the bottom to 7-9 at the top of the profile.

The accretion $V$ factor in the RICO case is underestimated by the more moderate value of about $20 \%-30 \%$. As shown in Fig. 5, the underestimation occurs mostly for large accretion rates, which are more frequent in upper regions of clouds. At the lower levels of the clouds, the accretion rates are small to moderate, and as Fig. 5 shows, the bias is positive there. The $V$ factor is less than 1 in the lower half of the cloud where accretion rates are overestimated and more than 1 in upper levels where accretion rates are underestimated. Obviously, the upper part of the cloud has, on average, twice the volume of the lower part. A similar pattern of the $V$-factor profile exists for the TOGA COARE clouds as well.
For application purposes, it is useful to have analytical approximations to the $V$-factor profiles. In both cases, the $V$ factors can be very accurately approximated by analytical fits in the form of third-order polynomials:

$$
V_{\mathrm{au}}(z)=\sum_{i=0}^{3} A_{i} z^{i} ; \quad V_{\mathrm{accr}}(z)=\sum_{i=0}^{3} B_{i} z^{i} .
$$

Coefficients of the analytical fits $A_{i}$ and $B_{i}$ are presented in Table 1; the analytical fits are shown in Fig. 4 by dashed lines. The accuracy of the analytical fits is quite high with coefficients of determination (COD) $R^{2}$ in the 0.87-0.99 range.

Additional information about the errors caused when neglecting SGS variability can be gained when considering their dependence on the value of the conversion rate itself ${ }^{4}$ (Fig. 5). For autoconversion, the errors increase with the value of the rate; for instance, in the

\footnotetext{
${ }^{4}$ The errors in Figs. 5 and 6 are defined according to (2), where $A$ represents noPDF conversion rates.
} 
TABLE 1. Coefficients of analytical fits to autoconversion $\left(A_{i}\right)$ and accretion $\left(B_{i}\right)$ variability factors for RICO and TOGA COARE cases; $R^{2}$ is the square of correlation coefficient also referred to as COD.

\begin{tabular}{|c|c|c|c|c|c|c|c|c|c|c|}
\hline & \multicolumn{5}{|c|}{ Autoconversion } & \multicolumn{5}{|c|}{ Accretion } \\
\hline & $A_{0}$ & $A_{1}$ & $A_{2}$ & $A_{3}$ & $R^{2}$ & $B_{0}$ & $B_{1}$ & $B_{2}$ & $B_{3}$ & $R^{2}$ \\
\hline RICO & 2.96 & 0.82 & -1.22 & 0.34 & 0.97 & 0.54 & -0.80 & 0.90 & -0.18 & 0.99 \\
\hline TOGA & 7.10 & -6.78 & 3.12 & -0.34 & 0.87 & -0.13 & 0.72 & -0.12 & 0.006 & 0.96 \\
\hline
\end{tabular}

RICO case, the small autoconversion rates have the errors in the $-65 \%$ to $-50 \%$ range, while the large autoconversion rates have also the largest errors (up to $-80 \%$ ). The correlation between autoconversion bias and the value of autoconversion rate may be explained by the fact that autoconversion rate is inversely proportional to the cloud drop concentration [(3)]; that is, they are larger in areas with small $N_{c}$. The latter are most commonly found in upper portions of the clouds, where updrafts and, consequently, collection are at maximum. These areas also have the largest cloud microphysical variability and, therefore, the largest bias when SGS variability is neglected.

In the TOGA COARE case, autoconversion errors also tend to increase with the value of the rate but, in general, display larger scatter, and the rate of increase, on average, is steeper compared to RICO.

For accretion, in the RICO case, the small accretion rates are positively biased (up to $+120 \%$ ), while the moderate to strong accretion rates have consistent negative bias (on the order of $-25 \%$ to $-40 \%$ ). The TOGA COARE accretion errors have larger scatter but show a pattern similar to the RICO case; that is, they are positive for small rates and negative for large accretion rates. For rates in the middle, the accretion errors show a large scatter, and their sign is not uniquely defined by the value of the rate.

In the model equations, both autoconversion and accretion act simultaneously, so it is useful to analyze the effect of their sum-the total conversion rate shown in Fig. 6. The bias of the total rate essentially follows the bias of the accretion rate; the latter is generally larger than autoconversion, especially when clouds are at mature stage and precipitation is already fully developed. Specifically, the bias in the RICO case is negative for rates larger than $0.7 \times 10^{-7} \mathrm{~s}^{-1}$. In the TOGA COARE case, only the rates larger than $0.4 \times 10^{-6} \mathrm{~s}^{-1}$ have a consistent negative bias; for the small, moderate, and a significant portion of the large rates, the bias may be either positive or negative, although the negative bias points clearly outnumber the positive ones. Nevertheless, the conclusion, that correcting for SGS variability should lead to enhancement of precipitation, cannot be warranted based on Fig. 6 results only. This is because one needs to consider the fact that when total (underestimated) conversion rate is added to the rainwater mixing ratio $q_{r}$, it is simultaneously subtracted from the cloud water mixing ratio $q_{c}$. As a result, the positive bias in $q_{c}$ may counterbalance the negative bias in $q_{r}$.
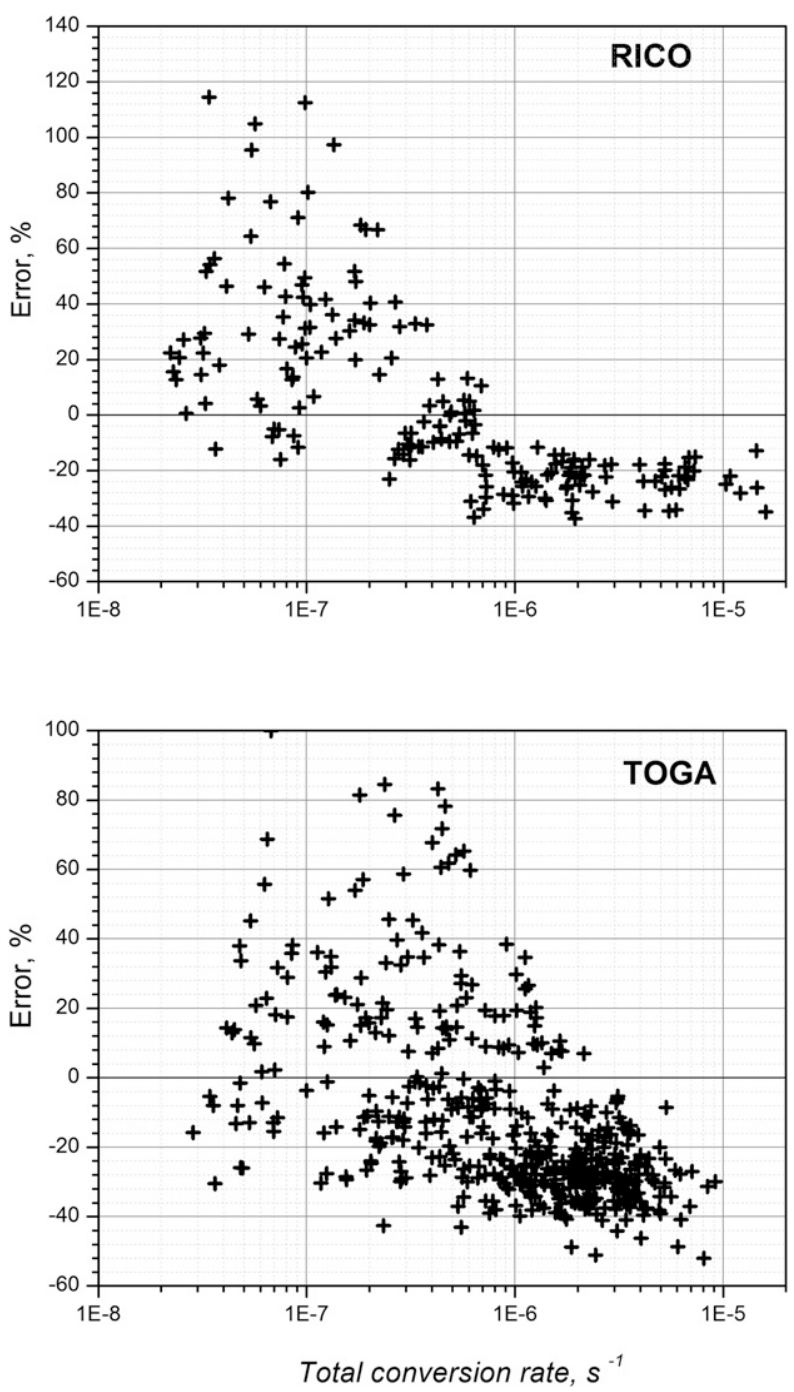

FIG. 6. The total conversion rate errors as a function of the rate value in (top) RICO and (bottom) TOGA COARE simulations. 
Consequently, the overall effect of neglecting variability needs to be investigated in numerical simulations.

The $V$ factor is designed for application and, strictly speaking, needs to be tested in a mesoscale model. A complete investigation of the precipitation effect of SGS variability in a mesoscale setting is a separate task that requires an effort beyond the scope of this study. However, the precipitation effect of SGS variability in a mesoscale simulation may be obscured by feedbacks and multiscale interactions between various mesoscale processes. A much easier and more focused study can be done in the LES setting where sensitivity experiments can evaluate if the conversion rates corrected by the $V$ factor substantially affect the precipitation process. If the tests show only a small effect on precipitation, then it would mean that inhomogeneity is of minor importance, and its accounting in mesoscale models may be unwarranted. Our tests, however, do show a large effect on precipitation; they also evaluate separate contributions from autoconversion and accretion, thus confirming the need for further testing in mesoscale models.

\section{Effect of inhomogeneity on precipitation}

The effect on precipitation of the conversion rates amended by $V$ factors was evaluated in RICO and TOGA COARE LESs. Three experiments were conducted in the RICO case: in the first, only the autoconversion rate was amended by including the $V$ factor; in the second, only accretion was amended; and in the third, both rates were amended simultaneously. In the TOGA case, because of computer time limitations, only one simulation was performed with both rates amended. These simulations were compared with the original benchmark simulations with no $V$ factors included.

Figure 7 shows the evolution of domain-averaged accumulated surface precipitation. In the RICO case, amending the autoconversion rate significantly increased surface precipitation-by about $38 \%$ from 4 to $24 \mathrm{~h}$. The amended accretion rates also increased surface precipitation but to a much lesser degree, mainly because of the already mentioned nearly linear dependence of accretion rate on its parameters. The synergetic effect of both rates amended together also increased precipitation; however, adding accretion appears at times (e.g., from 17 to 20 h; Fig. 7) to negate the positive effect of autoconversion. This is most likely due to negative feedbacks between $q_{r}$ and $q_{c}$ mentioned in previous section.

In the TOGA COARE simulation (bottom panel in Fig. 7), the inhomogeneity is much larger; therefore, there is a much more significant increase in surface precipitation - by about $75 \%$ over only $8 \mathrm{~h}$ of rain at the
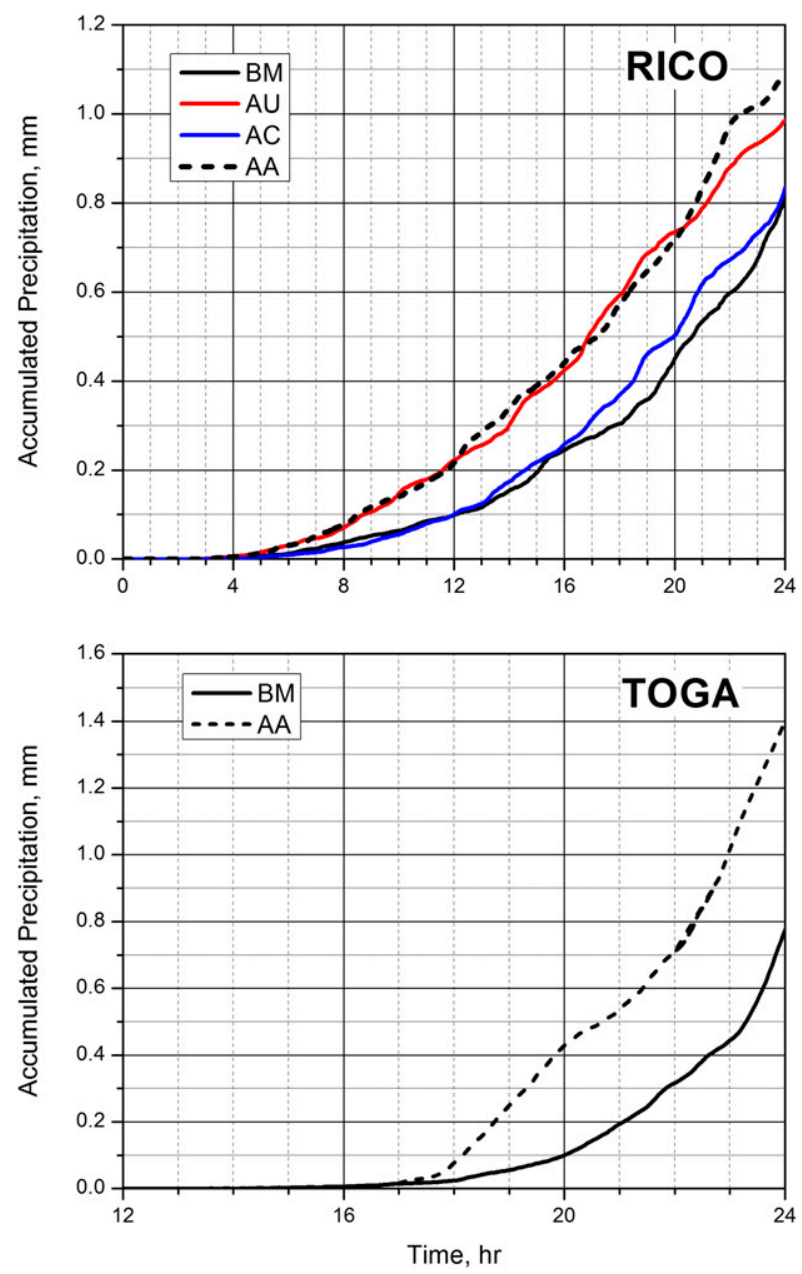

FIG. 7. Effect of accounting for inhomogeneity in microphysical conversion rates on accumulated surface precipitation in (top) RICO and (bottom) TOGA COARE simulations. Including $V$ factor 1) only in the autoconversion rate (red line: AU), 2) only in the accretion rate (blue line: AC), and 3) autoconversion and accretion together (dashed black line: AA). Solid black line represents the benchmark case (BM) with no $V$ factors added.

surface (from 16 to $24 \mathrm{~h}$ ). These results point to a possible quite substantial precipitation enhancement in mesoscale models when SGS variability is accounted for in conversion rates. In mesoscale settings, as was already mentioned in previous section, the quantitative effect needs to be reevaluated by considering all feedbacks and interactions between microphysical and multiscale thermodynamical processes.

\section{Conclusions}

Following the approach previously developed by KM14 and KM16, we evaluated different formulations of joint probability distribution functions (JPDFs) obtained from 
LES numerical experiments initialized with data from field projects investigating shallow cumulus (RICO) and cumulus congestus (TOGA COARE) convective clouds. We showed that inhomogeneity in both cloud types can be quantified by "generic" JPDFs, which are obtained using datasets from the entire simulation time cycle; that is, they represent the most general inhomogeneity characteristic of each cloud type.

Our results show that generic JPDFs do not significantly degrade the accuracy of JPDFs, which depend on time and, thus, change with the ambient conditions during evolution of the simulated cloud system. The advantage of using generic JPDFs is that they can be a priori integrated, yielding a one-dimensional variability factor ( $V$ factor). We found that the $V$ factor can be quite accurately approximated by an analytical function in the form of a third-order polynomial. The latter can be easily implemented into a mesoscale model to account for cloud inhomogeneity.

In the more general case when $V$ factors depend on ambient conditions, it is possible to derive and analyze a set of one-dimensional profiles of $V$ factors corresponding to different ambient parameters. Finding functional dependencies of such one-dimensional $V$ factors on mesoscale prognostic variables can be a much easier task than finding closure schemes for the twodimensional JPDFs.

Other findings include the quantification of the inhomogeneity bias dependence on microphysical conversion rates. We showed that, when inhomogeneity is neglected, the autoconversion rate is underestimated in the whole range of microphysical parameters by a factor of 2.5-3 for the RICO case. In the TOGA COARE case, the underestimation is even more significant-by a factor of 3-9 depending on the vertical level. The accretion rate bias quantitatively is smaller, but its sign depends on the cloud vertical level: it is negative in the lower part of the cloud and positive at the upper levels.

We also evaluated the effect of augmenting conversion rates by the $V$ factors on precipitation formation. Over the 24-h LES sensitivity experiments, the surface precipitation in the RICO case has substantially increased when $V$ factors were accounted for in conversion rates. In the TOGA case, the increase was even more significant. These increases, however, were smaller than biases in conversion rates, reflecting the fact that amended conversion rates nonlinearly affect the precipitation process, in part because of negative feedbacks between rain and cloud water mentioned in section 5 .

The sensitivity experiments revealed that most of the increase in precipitation was due to the augmented autoconversion rates, while the effect of accretion rate modifications was much less significant, primarily because of the nearly linear dependence of the accretion rate on its parameters. The large sensitivity to autoconversion clearly demonstrates the importance of its most accurate formulation in numerical models.

Finally, we would like to emphasize that conclusions of this study were based on LESs using initial conditions from the RICO and TOGA COARE field projects. To realistically quantify the effects of inhomogeneity on precipitation, we need more long-term case studies in mesoscale model settings that will include many feedbacks between microphysics and regional mesoscale dynamics.

Acknowledgments. This investigation was supported by the ONR Grants N00014-11-10439 and N00014-16-1-2487. The analysis results of conversion rate errors in shallow cumulus clouds were presented at the 2016 EMS meeting and published in Advances in Science and Research (Kogan 2017). The computing for this project was performed at the University of Oklahoma Supercomputing Center for Education and Research (OSCER). I would like to express my gratitude to the anonymous reviewers for their constructive comments.

\section{APPENDIX}

\section{Sensitivity of JPDFs to the Size of the LES Horizontal Domain}

In our approach, the JPDFs are derived from LES data in an integration domain whose horizontal size mimics the size of a mesoscale model grid. In the RICO simulation, the domain had $256 \times 256$ grid points using a horizontal resolution of $100 \mathrm{~m}$; this corresponded to a mesoscale grid of $25.6 \mathrm{~km}$. The mesoscale models, however, use a wide range of grid sizes: from 5 to $50 \mathrm{~km}$. The question then is whether JPDFs obtained in one domain size can be applied to other domain sizes. To answer, we evaluated sensitivity of conversion rate errors to the choice of the horizontal domain size used to derive JPDFs. We conducted four sensitivity experiments in domains with $64 \times 64,128 \times 128,256 \times 256$, and $512 \times 512$ grid points using the same horizontal resolution of $100 \mathrm{~m}$. These domains, thus, correspond to mesoscale grids of $6.4,12.8,25.6$, and $51.2 \mathrm{~km}$, respectively. The different domains in Fig. A1 are labeled as Dom64, Dom128, Dom256, and Dom512 with corresponding JPDF labeled as jpdf64, jpdf128, jpdf256, and jpdf512.

Fig. A1 shows cumulative distribution of autoconversion (Figs. A1a-d) and accretion (Figs. A1e-h) rate errors when different JPDFs are applied to domains of different sizes 

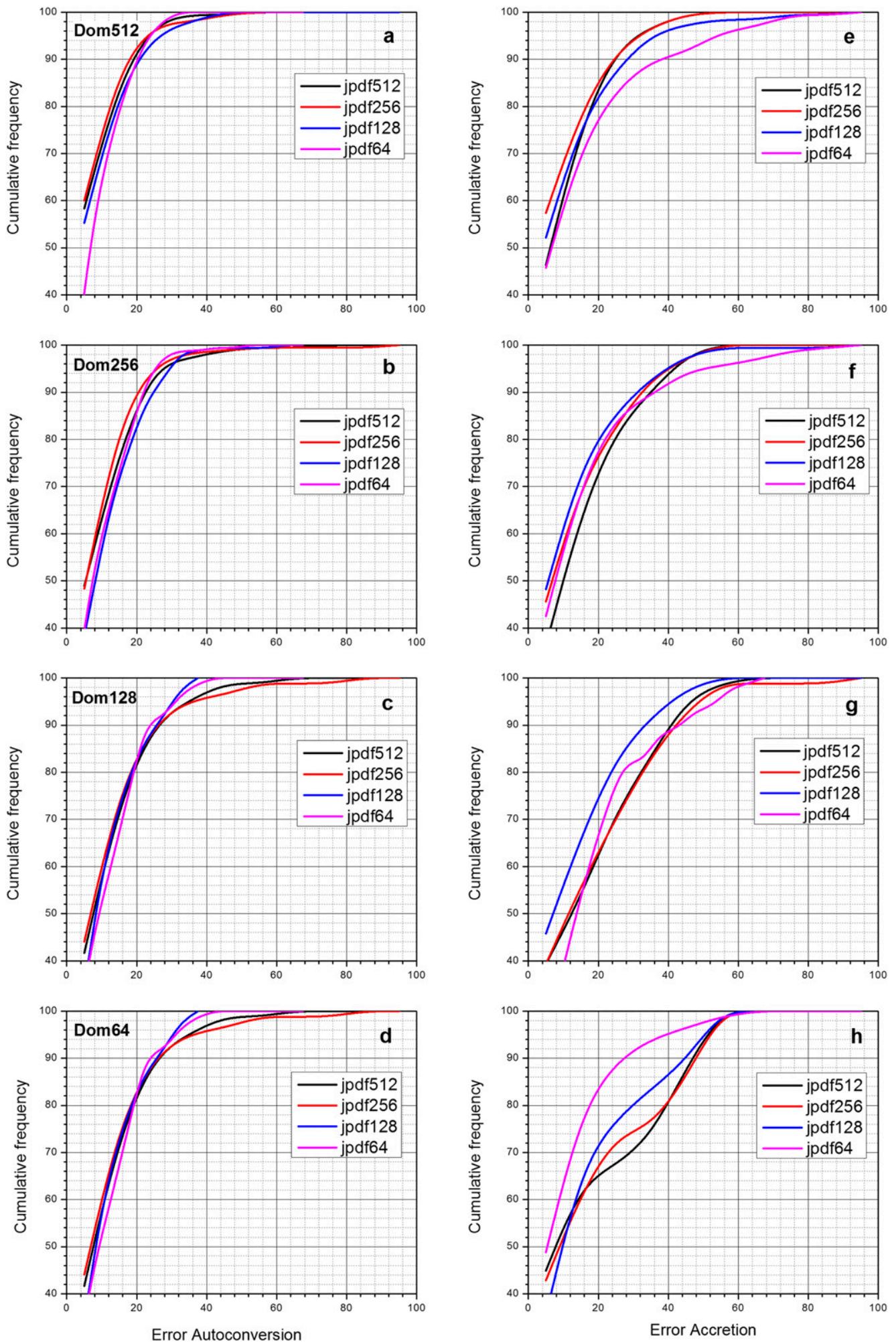

FIG. A1. Cumulative distributions of errors for (a)-(d) autoconversion and (e)-(h) accretion rates. Domain horizontal size decreases from (top) 51.2 (Dom512) to (bottom) $6.4 \mathrm{~km}$ (Dom64). Different colors in each panel refer to JPDFs obtained from datasets of different sizes. The largest dataset from Dom512 yielded the JPDF denoted jpdf512 (black); the smallest dataset from Dom64 correspondingly yielded jpdf64 (magenta). See appendix text for more explanation. 
(for simplicity, we consider only the absolute value of the errors).

The following conclusions can be made based on these sensitivity tests:

1) The autoconversion rates are less sensitive to the choice of JPDF than accretion rates.

2) The smallest errors, as well as the smallest sensitivity to the JPDF choice, are in large domains. Obviously, larger domains provide larger datasets, which can reproduce variability of the full range of clouds in the cloud system.

3) The JPDFs derived from larger, statistically more robust datasets (jpdf512 and jpdf256) produce the smallest errors when applied to large domains (Dom512 and Dom256). Both of these domains include the full range of RICO clouds, as trade wind shallow cumulus clouds normally do not exceed $7 \mathrm{~km}$ in size (e.g., Zhao and Di Girolamo 2007). Our results (not shown here) demonstrated that major cloud system properties (cloud cover, time series of surface accumulated precipitation, mean TKE) are very similar in Dom512 and Dom256 simulations. The results are less accurate in smaller domains (Dom 128 and Dom64). The smallest domain (Dom64) corresponds to a "gray zone"; that is, its size falls in between the cloud-resolving model (CRM) and mesoscale model grid size. As Fig. A1 shows, the smallest errors in Dom64 and Dom128 cases are produced when "native" JPDFs are used, that is, the JPDFs derived from the same domain where they are applied. The choice of native JPDF is especially important for minimizing errors in the accretion rate (Figs. A1g,h). However, as was shown in section 5 , because of the nearly linear formulation of the accretion rate, its effect on precipitation is much less significant. In summary, our results show that JPDFs derived from large datasets (Dom256, Dom512) can be applied for mesoscale grids in the range from about 12 to $50 \mathrm{~km}$; for smaller grids, the results are more accurate when native JPDFs are employed.

\section{REFERENCES}

Cheng, A., and K.-M. Xu, 2006: Simulation of shallow cumuli and their transition to deep convective clouds by cloud-resolving models with different third-order turbulence closures. Quart. J. Roy. Meteor. Soc., 132, 359-382, https://doi.org/10.1256/ qj.05.29.

— for simulation of drizzling boundary layer clouds. J. Atmos. Sci., 66, 2317-2334, https://doi.org/10.1175/2009JAS2944.1.

Cheng, C.-P., and R. A. Houze Jr., 1979: The distribution of convective and mesoscale precipitation in GATE radar echo patterns. Mon. Wea. Rev., 107, 1370-1381, https://doi.org/ 10.1175/1520-0493(1979)107<1370:TDOCAM>2.0.CO;2.
Godfrey, J. S., R. A. Houze Jr., R. H. Johnson, R. Lukas, J.-L. Redelsperger, A. Sumi, and R. Weller, 1998: Coupled OceanAtmosphere Response Experiment (COARE): An interim report. J. Geophys. Res., 103, 14395-14450, https://doi.org/ 10.1029/97JC03120.

Golaz, J.-C., V. E. Larson, and W. R. Cotton, 2002: A PDF-based model for boundary layer clouds. Part I: Method and model description. J. Atmos. Sci., 59, 3540-3551, https://doi.org/ 10.1175/1520-0469(2002)059<3540:APBMFB>2.0.CO;2.

Khairoutdinov, M., and Y. Kogan, 1999: A large eddy simulation model with explicit microphysics: Validation against aircraft observations of a stratocumulus-topped boundary layer. J. Atmos. Sci., 56, 2115-2131, https://doi.org/10.1175/15200469(1999)056<2115:ALESMW >2.0.CO;2.

$\longrightarrow$, and - 2000: A new cloud physics parameterization in a large-eddy simulation model of marine stratocumulus. Mon. Wea. Rev., 128, 229-243, https://doi.org/10.1175/1520-0493 (2000)128<0229:ANCPPI >2.0.CO;2.

__ , and D. A. Randall, 2003: Cloud resolving modeling of the ARM summer 1997 IOP: Model formulation, results, uncertainties, and sensitivities. J. Atmos. Sci., 60, 607-625, https://doi.org/10.1175/ 1520-0469(2003)060<0607:CRMOTA > 2.0.CO;2.

Kogan, Y., 2013: A cumulus cloud microphysics parameterization for cloud-resolving models. J. Atmos. Sci., 70, 1423-1436, https://doi.org/10.1175/JAS-D-12-0183.1.

_ 2017: LES study of microphysical variability bias in shallow cumulus. Adv. Sci. Res., 14, 103-107, https://doi.org/10.5194/ asr-14-103-2017.

— rameterization for shallow cumulus clouds. J. Atmos. Sci., $\mathbf{7 1}$, 1070-1089, https://doi.org/10.1175/JAS-D-13-0193.1.

— variability in cumulus congestus clouds. J. Atmos. Sci., 73 , 167-184, https://doi.org/10.1175/JAS-D-15-0129.1.

_, M. P. Khairoutdinov, D. K. Lilly, Z. N. Kogan, and Q. Liu, 1995: Modeling of stratocumulus cloud layers in a large eddy simulation model with explicit microphysics. J. Atmos. Sci., 52, 2923-2940, https://doi.org/10.1175/1520-0469(1995)052<2923: MOSCLI $>2.0 . \mathrm{CO} ; 2$.

_ D. B. Mechem, and K. Choi, 2012: Effects of sea-salt aerosols on precipitation in simulations of shallow cumulus. J. Atmos. Sci., 69, 463-483, https://doi.org/10.1175/JAS-D-11-031.1.

Lappen, C.-L., and D. A. Randall, 2001: Toward a unified parameterization of the boundary layer and moist convection. Part I: A new type of mass-flux model. J. Atmos. Sci., 58, 2021-2036, https://doi.org/10.1175/1520-0469(2001)058<2021: TAUPOT $>2.0 . \mathrm{CO} ; 2$.

Larson, V. E., and J.-C. Golaz, 2005: Using probability density functions to derive consistent closure relationships among higher-order moments. Mon. Wea. Rev., 133, 1023-1042, https://doi.org/10.1175/MWR2902.1.

— R. Wood, P. R. Field, J.-C. Golaz, T. H. Vonder Harr, and W. R. Cotton, 2001a: Systematic biases in the microphysics and thermodynamics of numerical models that ignore subgridscale variability. J. Atmos. Sci., 58, 1117-1128, https://doi.org/ 10.1175/1520-0469(2001)058<1117:SBITMA $>2.0$.CO;2.

$-,-, \ldots,-, \ldots$, and,$- 2001 \mathrm{~b}$ : Small-scale and mesoscale variability of scalars in cloud boundary layers: Onedimensional probability density functions. J. Atmos. Sci., 58 , 1978-1994, https://doi.org/10.1175/1520-0469(2001)058<1978: $\mathrm{SSAMVO}>2.0 . \mathrm{CO} ; 2$.

_ J.-C. Golaz, and W. R. Cotton, 2002: Small-scale and mesoscale variability in cloudy boundary layers: Joint probability 
density functions. J. Atmos. Sci., 59, 3519-3539, https://doi.org/ 10.1175/1520-0469(2002)059<3519:SSAMVI $>2.0 . C O ; 2$.

Lee, S., B. H. Kahn, and J. Teixeira, 2010: Characterization of cloud liquid water content distributions from CloudSat. J. Geophys. Res., 115, D20203, https://doi.org/10.1029/ 2009JD013272.

Lewellen, W. S., and S. Yoh, 1993: Binormal model of ensemble partial cloudiness. J. Atmos. Sci., 50, 1228-1237, https://doi.org/ 10.1175/1520-0469(1993)050<1228:BMOEPC $>2.0 . C O ; 2$.

Liu, C., and E. J. Zipser, 2009: "Warm rain" in the tropics: Seasonal and regional distributions based on $9 \mathrm{yr}$ of TRMM data. J. Climate, 22, 767-779, https://doi.org/10.1175/ 2008JCLI2641.1.

Mechem, D. B., and A. J. Oberthaler, 2013: Numerical simulation of tropical cumulus congestus during TOGA COARE. J. $A d v$. Model. Earth Syst., 5, 623-637, https://doi.org/10.1002/jame.20043.

Pincus, R., and S. A. Klein, 2000: Unresolved spatial variability and microphysical process rates in large-scale models. J. Geophys. Res., 105, 27 059-27 065, https://doi.org/10.1029/ 2000JD900504.

Price, J. D., and R. Wood, 2002: Comparison of probability density functions for total specific humidity and saturation deficit humidity, and consequences for cloud parameterization. Quart. J. Roy. Meteor. Soc., 128, 2059-2072, https://doi.org/ 10.1256/003590002320603539.

Rauber, R. M., and Coauthors, 2007: Rain in shallow cumulus over the ocean: The RICO campaign. Bull. Amer. Meteor. Soc., 88, 1912-1928, https://doi.org/10.1175/BAMS-88-12-1912.

Seifert, A., and K. D. Beheng, 2001: A double-moment parameterization for simulating autoconversion, accretion and selfcollection. Atmos. Res., 59-60, 265-281, https://doi.org/ 10.1016/S0169-8095(01)00126-0.

Smolarkiewicz, P. K., and W. W. Grabowski, 1990: The multidimensional positive definite advection transport algorithm: Nonoscillatory option. J. Comput. Phys., 86, 355-375, https:// doi.org/10.1016/0021-9991(90)90105-A.

Stephens, G. L., and N. B. Wood, 2007: Properties of tropical convection observed by millimeter-wave radar systems. Mon. Wea. Rev., 135, 821-842, https://doi.org/10.1175/ MWR3321.1.

vanZanten, M. C., and Coauthors, 2011: Controls on precipitation and cloudiness in simulations of trade-wind cumulus as observed during RICO. J. Adv. Model. Earth Syst., 3, M06001, https://doi.org/10.1029/2011MS000056.

Wang, S., and B. Stevens, 2000: Top-hat representation of turbulence statistics in cloud-topped boundary layers: A large eddy simulation study. J. Atmos. Sci., 57, 423-441, https://doi.org/ 10.1175/1520-0469(2000)057<0423:THROTS $>2.0$.CO;2.

Wood, R., P. R. Field, and W. R. Cotton, 2002: Autoconversion rate bias in stratiform boundary layer cloud parameterizations. Atmos. Res., 65, 109-128, https://doi.org/10.1016/S01698095(02)00071-6.

Xu, K.-M., and D. A. Randall, 1996: Evaluation of statistically based cloudiness parameterizations used in climate models. J. Atmos. Sci., 53, 3103-3119, https://doi.org/ 10.1175/1520-0469(1996)053<3103:EOSBCP > 2.0.CO;2.

Zhao, G., and L. Di Girolamo, 2007: Statistics on the macrophysical properties of trade wind cumuli over the tropical western Atlantic. J. Geophys. Res., 112, D10204, https:// doi.org/10.1029/2006JD007371. 